Research Article

\title{
Robust Tracking and Cruise Control of a Class of Robotic Systems
}

\author{
Ricardo Cuesta, ${ }^{1,2}$ Joaquín Alvarez, ${ }^{1}$ and Manuel Miranda ${ }^{2}$ \\ ${ }^{1}$ Departamento de Electrónica y Telecomunicaciones, CICESE, 22860 Ensenada, BCN, Mexico \\ ${ }^{2}$ Facultad de Ingeniería, Arquitectura y Diseño, UABC, 22860 Ensenada, BCN, Mexico \\ Correspondence should be addressed to Ricardo Cuesta; jcuesta@cicese.mx
}

Received 31 October 2014; Revised 20 January 2015; Accepted 21 January 2015

Academic Editor: Luis Rodolfo Garcia Carrillo

Copyright (c) 2015 Ricardo Cuesta et al. This is an open access article distributed under the Creative Commons Attribution License, which permits unrestricted use, distribution, and reproduction in any medium, provided the original work is properly cited.

\begin{abstract}
This paper presents a controller for a class of robotic systems, based on a first-order sliding mode with a particular noninvariant, nonconnected surface. With this control it is possible to regulate the position such that the velocity remains, as long as possible, at a specified value until the system is close to the desired position. The properties inherited from the sliding modes make the control exhibit a high robustness to external perturbations and low sensitivity to system parameter variations. It is shown that the desired speed is reached in a finite time and the system converges exponentially to the desired position. This controller can be applied to systems described by a classical model of a fully actuated, $n$-DOF mechanical system, which could be decoupled via a preliminary decoupling control. To illustrate the theoretical results, the proposed control technique is applied to a Cartesian robot, simulated numerically. Moreover, to show the effectiveness of this strategy, some physical experiments on a rotational (mechanical) device were performed.
\end{abstract}

\section{Introduction}

Sliding mode control (SMC) is a nonlinear control technique that, by using a discontinuous control signal, forces the system to move along a previously designed switching surface [1]. SMC has attired a great interest because its implementation is simple and it exhibits high robustness to external perturbations and exhibits low sensitivity to parameter variations of the system. These advantages has made SMC very popular in applications, including electrical, mechanical, chemical, industrial, civil, military, aeronautical, and aerospace engineering [2], among others.

Usually, the sliding surface is represented by a linear function of the state. A typical design makes this surface invariant, containing the control target which is made asymptotically stable with a suitable choice of the controller parameters. This has been enough to regulate, for example, the position of mechanical devices. On the other hand, there exist control algorithms that provide a satisfactory performance to regulate the velocity, for example, keeping a constant velocity in a motor shaft $[3,4]$.

Combination of both goals, that is, attaining a constant state while keeping a constant velocity in the most part of the displacement stage, is, however, a more complicated goal analyzed less frequently. This double control objective is easy to associate in aeronautics, where the aircraft arrives to a point $B$ from a point $A$ using three stages: takeoff (velocity increases until the aircraft reaches the desired constant velocity), cruise speed (keeping the aircraft in the desired constant velocity), and landing (velocity decreases until the aircraft reaches the final destination or desired position). Some advantages of cruise speed in aircraft are as follows: there is a high decrease in fuel consumption, the aerodynamics levels produce less pressure or less force under the thrust resistance, the motors work in optimal levels of temperature, pressure, and wear, increasing the motor life, because they are not submitted to high efforts [5], and so on. These characteristics are also present in land vehicles, submarine vehicles, and ships.

There are many other applications where it is useful that the vehicle kept a constant velocity before arriving to a fixed desired position. Some examples in aerial transport are helicopters, planes, satellites, spaceships, missiles and, in recent years, unmanned aerial vehicles (UAV). Moreover, there are ships, trains, submarines, telescopes, elevators, robots, mobile robots, conveyors, corrosive liquid transportation, 
and molten metal transportation that do not admit abrupt changes in speed.

There are many works, like [6-10], where the main control objective is to maintain a desired altitude position in planes, satellites, spaceships, missiles, and UAV, respectively, without imposing a speed behavior. In other works like [11-13], the main control objective is to keep a desired velocity (cruise speed) in ships, trains, and telescopes, respectively, without considering a final position strategy.

In the present paper we propose a control law, based on a first-order sliding mode with a particular noninvariant, nonconnected surface, for a class of robotic systems. The shape of this surface produces indirectly a system behavior which switches from a first- to a second-order sliding mode. First, the control forces the system to attain the noninvariant surface, keeping a constant, desired speed while the system moves on this surface. Later, once the system is close to the desired position, it leaves the sliding surface to converge to this position through a second-order sliding mode. With this proposed control it is possible to solve the combined objectives described previously, that is, position regulation, such that the velocity remains, as long as possible, at a specified value until the system is close to the desired position.

The properties inherited from the sliding modes make the proposed control exhibit a high robustness to external perturbations and low sensitivity to system parameter variations. It is shown that the desired velocity is reached in a finite time and the system converges exponentially to the desired position. This control algorithm can be applied to robotic systems described by a classical model of a fully actuated, $n$-DOF mechanical system, which could be decoupled via a preliminary decoupling control.

To illustrate the theoretical results, the proposed control technique is applied to a Cartesian robot, simulated numerically. Moreover, to show the effectiveness of this strategy, some physical experiments on a rotational (mechanical) device were performed.

The paper is organized as follows. In Section 2, the basic system and controller are described. Section 3 presents the main result and the formal demonstration of stability. Section 4 illustrates the performance of the proposed controller through numerical and experimental implementations. Finally, some conclusions and remarks are presented in Section 5.

\section{A Controlled Mechanical System}

Let us consider an $n$-DOF mechanical system described by

$$
\mathbf{M}(q) \ddot{q}+\mathbf{C}(q, \dot{q}) \dot{q}+g(q)=\tau
$$

where $\mathbf{M}(q)$ is the $n \times n$ symmetric, positive definite inertia matrix for all $q \in \mathbb{R}^{n}, \mathbf{C}(q, \dot{q})$ is the $n \times n$ matrix of centrifugal and Coriolis forces, $g(q) \in \mathbb{R}^{n}$ is the vector of gravitational forces, and $\tau \in \mathbb{R}^{n}$ is the vector of external forces. If

$$
\tau=\mathbf{M}(q) u+[\mathbf{C}(q, \dot{q})-\mathbf{M}(q) \mathbf{B}] \dot{q}+g(q)=\mathbf{M}(q) u+u_{0},
$$

where $u_{0}=[\mathbf{C}(q, \dot{q})-\mathbf{M}(q) \mathbf{B}] \dot{q}+g(q), u$ is a new control input, and $\mathbf{B}$ is a diagonal, positive definite $n \times n$ matrix, and then, after the application of this control input, system (1) becomes

$$
\ddot{q}=-\mathbf{B} \dot{q}+u \text {. }
$$

This system can be seen as a set of decoupled, second-order systems with the form

$$
\ddot{q}_{i}=-b_{i} \dot{q}_{i}+u_{i}
$$

Obviously, a similar form can be obtained when there is no gravity effect; that is, $g=\mathbf{0}$, and $\mathbf{C}=\mathbf{B}$.

In a general and simple way, airplanes, projectiles, ships, and terrestrial vehicles can be modeled like a simple mass in movement, using Newton's second law of motion, with an opposition force caused by the viscous friction, which is proportional to the mass velocity. This is a particular case of (1), which can be easily transformed to (4), for which we can employ the following state equations:

$$
\begin{gathered}
\dot{x}_{1}=x_{2}, \\
\dot{x}_{2}=-b x_{2}+u,
\end{gathered}
$$

where, for simplicity, we have dropped the index $i, x_{1}=q_{i}$, and $x_{2}=\dot{q}_{i}$.

Equation (5) can describe also the dynamical behavior of other mechanical devices, for example, a motor shaft with viscous friction, where the moment of inertia is used instead of the mass, and the position and velocity are angular variables. This behavior is similar in propellers, turbines, drilling machines, and other similar torsional mechanisms. In summary, system (5) describes a fundamental dynamics of a wide variety of mechanical systems.

In what follows we propose a control law for system (5) and analyze the stability properties of the closed-loop system, and then we apply this control strategy to several systems like (1).

Let us consider the sign function, which is defined in the Filippov sense as

$$
\operatorname{sign}(m)= \begin{cases}-1, & m<0 \\ \eta, & m=0 \\ +1, & m>0\end{cases}
$$

where $\eta \in[-1,1]$. Moreover, define a discontinuous function, denoted by $\operatorname{sgn}(m)$ and described by

$$
\operatorname{sgn}(m)= \begin{cases}-1, & m<0 \\ 0, & m=0 \\ +1, & m>0\end{cases}
$$

Now, consider a control law, applied to system (5), given by

$$
u=-\alpha \operatorname{sign}(\sigma(x))
$$

where $\sigma(x)$ is defined as

$$
\sigma(x)=\beta \operatorname{sgn}\left(x_{1}\right)+x_{2},
$$


where $x=\left(x_{1}, x_{2}\right)^{T}$ and $\alpha$ and $\beta$ are positive real constants. The closed-loop system, formed by the system (5) and the control law (8), is given by

$$
\begin{gathered}
\dot{x}_{1}=x_{2}, \\
\dot{x}_{2}=-b x_{2}-\alpha \operatorname{sign}(\sigma(x)) .
\end{gathered}
$$

The state space $\mathbb{R}^{2}$ is split in two disjoint sets, $\Sigma_{-}$and $\Sigma_{+}$, characterized by the sign of $\sigma(x)$, described by (see Figure 1)

$$
\begin{aligned}
& \Sigma_{-}=\left\{x \in \mathbb{R}^{2} \mid \sigma(x)<0\right\}, \\
& \Sigma_{+}=\left\{x \in \mathbb{R}^{2} \mid \sigma(x)>0\right\} .
\end{aligned}
$$

$\Sigma_{+}$and $\Sigma_{-}$(denoted as $\Sigma_{ \pm}$) are delimited by another region, denoted by $\Sigma_{0}$, which we call the commutation curve, given by

$$
\Sigma_{0}=\Sigma_{0-} \cup \Sigma_{00} \cup \Sigma_{0+},
$$

where

$$
\begin{aligned}
& \Sigma_{0-}=\left\{x \in \mathbb{R}^{2} \mid x_{1}<0, x_{2}=\beta\right\}, \\
& \Sigma_{00}=\left\{x \in \mathbb{R}^{2}\left|x_{1}=0,\right| x_{2} \mid \leq \beta\right\}, \\
& \Sigma_{0+}=\left\{x \in \mathbb{R}^{2} \mid x_{1}>0, x_{2}=-\beta\right\} .
\end{aligned}
$$

In the regions $\Sigma_{ \pm}$, the state equations (10) become

$$
\begin{gathered}
\dot{x}_{1}=x_{2}, \\
\dot{x}_{2}=-b x_{2}-\alpha_{s},
\end{gathered}
$$

where

$$
\alpha_{s}= \pm \alpha \quad \text { when } x \in \Sigma_{ \pm} .
$$

Note that system (14) is piece-wise linear, with a constant input $-\alpha_{s}$ in each region. Then the solution can be calculated as

$$
\begin{gathered}
x_{1}(t)=x_{1}\left(t_{k}\right)+\frac{1}{b^{2}}\left[1-\exp \left(-b\left(t-t_{k}\right)\right)\right]\left[b x_{2}\left(t_{k}\right)+\alpha_{s}\right] \\
-\frac{\alpha_{s}}{b}\left(t-t_{k}\right) \\
x_{2}(t)=\exp \left(-b\left(t-t_{k}\right)\right)\left[x_{2}\left(t_{k}\right)+\frac{\alpha_{s}}{b}\right]-\frac{\alpha_{s}}{b}
\end{gathered}
$$

for $t_{k} \leq t<t_{k+1}$, where $t_{k}$ is the time where the $k$ th commutation of $\operatorname{sign}(\sigma(x))$ occurs.

\section{Stability of the Closed-Loop System}

In this section we establish some conditions for the origin to be a global asymptotically stable equilibrium point of the controlled system (10). We show first that the control law (8) and (9) makes the system exhibit a sliding motion along the regions $\Sigma_{0-}$ and $\Sigma_{0+}$, such that the state approaches the $x_{2}$ axis with constant speed. Thereafter, once the state arrives at the $x_{2}$-axis, we will show that this control makes the system converge to the origin in an oscillatory way, generating a second-order sliding mode.

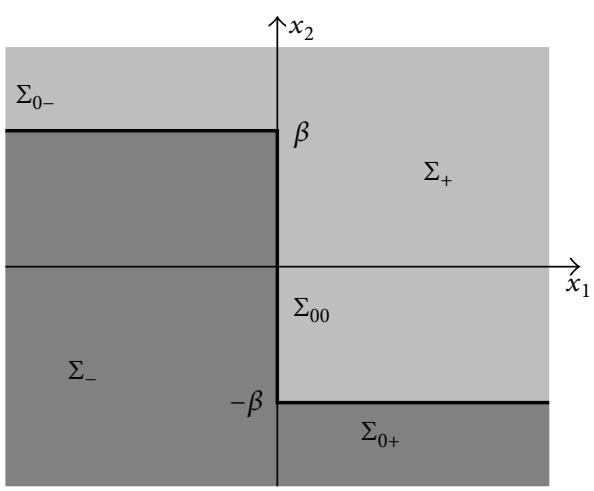

FIGURE 1: Regions generated by the commutation curve (9).

Lemma 1. For the controlled system (10), suppose that $\alpha>$ $b \beta>0$. Therefore, the system exhibits a sliding mode along the region $\Sigma_{0-}$ if $x_{1}(0)<0$ and $x_{2}(0) \approx \beta$ or along $\Sigma_{0+}$ if $x_{1}(0)>0$ and $x_{2}(0) \approx-\beta$, during a finite time interval.

Proof. To prove that $\Sigma_{0 \pm}$ are surfaces where a sliding motion may occur, it suffices to show that $\sigma(x) \dot{\sigma}(x)<0$ is satisfied for any point $x$ close enough to these surfaces. For $\Sigma_{0-}$ we have $x_{1}<0$; hence $\sigma(x)=-\beta+x_{2}$ and $\sigma \dot{\sigma}=-\sigma\left(b x_{2}+\alpha \operatorname{sgn}(\sigma)\right)$. Now, when $x_{2}>\beta>0$ we have $\sigma>0$, so $\sigma \dot{\sigma}<0$. In the same way, if $\sigma=-\beta+x_{2}<0$, then, when $x_{2} \leq 0 \sigma=-\beta+x_{2}<0$ and $\sigma \dot{\sigma}=-b x_{2} \sigma-\alpha|\sigma|<0$. Moreover, when $0<x_{2}<\beta$ then because $b \beta<\alpha$ we have $0<b x_{2}<b \beta<\alpha$; therefore $\sigma \dot{\sigma}=-\sigma\left(b x_{2}-\alpha\right)<0$. A similar analysis can be made for $\Sigma_{0+}$.

Finally, note that, once the system arrives at a point $\left(\bar{x}_{1}, \mp \beta\right)$ on the surface $\Sigma_{0 \pm}$, then a sliding mode is generated along these lines, directed toward the $x_{2}$-axis $\left(x_{1}=0\right)$, with a duration of $\left|\bar{x}_{1}\right| / \beta$ seconds.

Remark 2. Note that the region defined by

$$
G=\left\{\left(x_{1}, x_{2}\right) \in \mathbb{R}^{2}:\left|x_{2}\right| \leq \beta\right\}
$$

is attractive. This can be seen from (16) for the state $x_{2}$, which converges to $\bar{x}_{2}=\mp \alpha / b$ from $\Sigma_{ \pm}$. If $x_{2}\left(t_{0}\right)>\beta$, then $x\left(t_{0}\right) \in$ $\Sigma_{+}$and $\bar{x}_{2}=-\alpha / b<0$, and $x(t)$ arrives at $x_{2}=\beta$ at time $t=$ $t_{0}+(1 / b) \ln \left[\left(b x_{2}\left(t_{0}\right)+\alpha\right) /(b \beta+\alpha)\right]$. Similarly, if $x_{2}\left(t_{0}\right)<-\beta$, then $x(t)$ arrives at $x_{2}=-\beta$ at time $t=t_{0}+(1 / b) \ln \left[\left(b x_{2}\left(t_{0}\right)-\right.\right.$ $\alpha) /(b \beta-\alpha)]$ because $b \beta<\alpha$. Note also that $G$ is an invariant set; hence, any point in $\mathbb{R}^{2}$ outside $G$ arrives to this set in finite time and once it arrives, it remains there.

Let us define $\Sigma_{s}$ as the union of $\Sigma_{0-}$ and $\Sigma_{0+}$. This is the region where a sliding motion takes place, so

$$
\Sigma_{s}=\Sigma_{0-} \cup \Sigma_{0+} .
$$

Remark 3. It is straightforward to show that the sliding condition $\sigma \dot{\sigma}<0$ is satisfied for any point outside the commutation surface $\Sigma_{0}(12)$. However, not every solution $x(t)$ reaches the sliding surface $\Sigma_{s}$. This will be analyzed in what follows. 


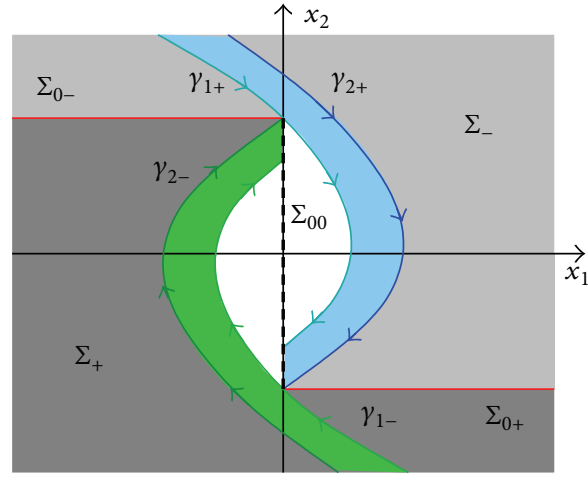

(a)

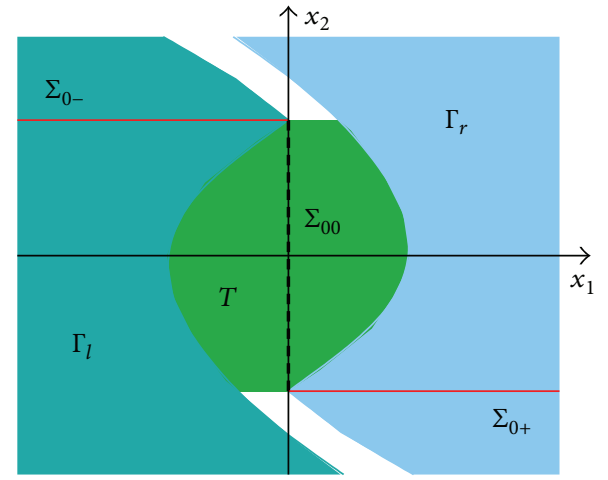

(b)

Figure 2: Basins of attraction of the sliding surfaces $\Sigma_{0 \mp}$.

Remark 4. Note that $\Sigma_{s}$ is nonconnected. It is also noninvariant, and any sliding orbit on this surface leaves the surface when the position $x_{1}$ reaches the zero-value. This is different to a classical sliding mode control design, where the sliding surface is invariant and connected and contains the control target (the origin in a suitable coordinate framework).

To prove that all orbits converge to the origin, let us consider two particular trajectory families, characterized with the aid of the next functions:

$$
\begin{aligned}
& \gamma_{+}(x)=b\left(b x_{1}+x_{2}-\beta\right)-\alpha \ln \frac{b x_{2}+\alpha}{b \beta+\alpha}, \quad x \in \Sigma_{+} \\
& \gamma_{-}(x)=b\left(b x_{1}+x_{2}+\beta\right)+\alpha \ln \frac{-b x_{2}+\alpha}{b \beta+\alpha}, \quad x \in \Sigma_{-} .
\end{aligned}
$$

Figure 2(a) shows several orbits for particular values of $\gamma_{ \pm}$. In this figure, $\gamma_{1 \pm}$ corresponds to $\gamma_{ \pm}(x)=0$ and $\gamma_{2 \pm}$ to $\gamma_{ \pm}(x)=$ $\mp 2 b \beta \mp \alpha \ln [(\alpha-b \beta) /(\alpha+b \beta)]>(<) 0$. We then have the next result.

Theorem 5. Suppose that $\alpha>b \beta>0$. Then the origin $0 \in$ $\mathbb{R}^{2}$ is a global, asymptotically stable equilibrium point of the controlled system (10) and (9).

Proof. Let us consider the trajectories

$$
\gamma_{1-}=\left\{x \in \mathbb{R}^{2}: \gamma_{-}(x)=0, x_{1} \geq 0, x_{2} \leq-\beta\right\}
$$

$$
\begin{aligned}
& \gamma_{2+} \\
& =\left\{x \in \mathbb{R}^{2}: \gamma_{+}(x)=-2 b \beta-\alpha \ln \left(\frac{\alpha-b \beta}{\alpha+b \beta}\right), \sigma(x)>0\right\},
\end{aligned}
$$

where $\gamma_{ \pm}(x)$ are given by (19) and $\sigma(x)$ by (9). Define the regions

$$
\Gamma_{1-}=\left\{x \in \mathbb{R}^{2}: \gamma_{-}(x) \geq 0, x_{1} \geq 0, x_{2} \leq-\beta\right\},
$$

$$
\begin{gathered}
\Gamma_{2+} \\
=\left\{x \in \mathbb{R}^{2}: \gamma_{+}(x) \geq-2 b \beta-\alpha \ln \left(\frac{\alpha-b \beta}{\alpha+b \beta}\right), \sigma(x)>0\right\}, \\
\Gamma_{r}=\Gamma_{1-} \cup \Gamma_{2+} .
\end{gathered}
$$

Note that $\Gamma_{r}$ is the region to the right of the particular trajectories $\gamma_{1-}$ and $\gamma_{2+}$ (the blue region of Figure 2(b)). It is straightforward to show that any trajectory starting in $\Gamma_{r}$ converges to the sliding region $\Sigma_{0+}$. In fact, only the trajectories starting in this region converge to this sliding surface. Similarly, regions $\Gamma_{1+}$ and $\Gamma_{2-}$ can be built from the trajectories

$$
\begin{gathered}
\gamma_{1+}=\left\{x \in \mathbb{R}^{2}: \gamma_{+}(x)=0, x_{1} \leq 0, x_{2} \geq \beta\right\}, \\
\gamma_{2_{-}}=\left\{x \in \mathbb{R}^{2}: \gamma_{-}(x)=2 b \beta+\alpha \ln \left(\frac{\alpha-b \beta}{\alpha+b \beta}\right), \sigma(x)<0\right\},
\end{gathered}
$$

as

$$
\begin{gathered}
\Gamma_{1+}=\left\{x \in \mathbb{R}^{2}: \gamma_{+}(x) \leq 0, x_{1} \leq 0, x_{2} \geq \beta\right\}, \\
\Gamma_{2-}=\left\{x \in \mathbb{R}^{2}: \gamma_{-}(x) \leq 2 b \beta+\alpha \ln \left(\frac{\alpha-b \beta}{\alpha+b \beta}\right), \sigma(x)<0\right\}, \\
\Gamma_{l}=\Gamma_{1+} \cup \Gamma_{2-},
\end{gathered}
$$

composing the region $\Gamma_{l}$ of all trajectories converging to the sliding surface $\Sigma_{0-}$ (see Figure 2(b)). Once the system arrives at $\Sigma_{s}$ it attains the region $\Sigma_{00}=\left\{\left(0, x_{2}\right) \in \mathbb{R}^{2}:\left|x_{2}\right| \leq \beta\right\}$ in finite time. Also, it is easy to show that any trajectory starting in the region $\mathbb{R}^{2} \backslash\left(\Gamma_{r} \cup \Gamma_{l}\right)$ does not attain the sliding surfaces $\Sigma_{0+}$ or $\Sigma_{0-}$ and arrives at the interval $\Sigma_{00}$ also in finite time.

In summary, any trajectory arrives at $\Sigma_{00}$ in finite time. Furthermore, all the trajectories coming from the sliding surfaces arrive at the point $(0, \pm \beta)$, and any other trajectory arrives at $\left(0, x_{2}\right)$, with $\left|x_{2}\right|<\beta$. Because the set $G(17)$ is invariant, once the system arrives at $\Sigma_{00}$ it stays in the region 
$T=\left\{\mathbb{R}^{2} \backslash\left(\Gamma_{r} \cup \Gamma_{l}\right)\right\} \cap G$ (dark green in Figure 2(b)), where an oscillatory motion is generated due to the commutation at $x_{1}=0$. Note that, for $x \in G, \operatorname{sign}(\sigma(x))=\operatorname{sign}\left(x_{1}\right)$. Therefore, the system dynamics can be expressed as

$$
\begin{gathered}
\dot{x}_{1}=x_{2}, \\
\dot{x}_{2}=-b x_{2}-\alpha \operatorname{sign}\left(x_{1}\right) .
\end{gathered}
$$

We can apply the tools developed elsewhere [14] to analyze the stability of the origin of system (24). For that, consider the Lyapunov function candidate

$$
V(x)=\frac{1}{2} x_{2}^{2}+\alpha\left|x_{1}\right|,
$$

whose derivative along the trajectories of system (24) is given by

$$
\dot{V}(x)=-b x_{2}^{2} \leq 0 .
$$

Then we conclude that the system converges to the $x_{1}$-axis $\left(x_{2} \rightarrow 0\right)$. By applying the extension of LaSalle theorem described in [14], it can be seen that the maximal invariant set inside $\left\{x \in \mathbb{R}^{2}: \dot{V}(x)=0\right\}$ is the origin. This can be also seen by analyzing the direction of vector fields at points $\left(x_{1}, \pm \epsilon\right)$, with $x_{1} \neq 0$ and $0<\epsilon \ll 1$. In consequence, the controlled system asymptotically converges to the origin.

We have the following corollaries, which are immediate consequences of Theorem 5 .

Corollary 6 (regulator). Consider the system described by

$$
\begin{gathered}
\dot{x}_{1}=x_{2}, \\
\dot{x}_{2}=-b x_{2}-\alpha \operatorname{sign}\left(\beta \operatorname{sgn}\left(x_{1}-x_{d}\right)+x_{2}\right),
\end{gathered}
$$

with $b, \alpha$, and $\beta$ positive real constant, and $x_{d} \in \mathbb{R}$ is a constant reference value. If $\alpha>b \beta>0$, then $x_{1} \rightarrow x_{d}$ and $x_{2} \rightarrow 0$ as $t \rightarrow \infty$.

Proof. Let us denote the error signals by $e_{1}=x_{1}-x_{d}$ and $e_{2}=x_{2}$. Then we have

$$
\begin{gathered}
\dot{e}_{1}=e_{2}, \\
\dot{e}_{2}=-b e_{2}-\alpha \operatorname{sign}\left(\beta \operatorname{sgn}\left(e_{1}\right)+e_{2}\right) .
\end{gathered}
$$

These equations have the form described in (10) and (9). By applying Theorem 5 we obtain that $e_{1} \rightarrow 0$ and $e_{2} \rightarrow 0$, so $x_{1} \rightarrow x_{d}$ and $x_{2} \rightarrow 0$ as $t \rightarrow \infty$.

Corollary 7 (tracking). Consider the system described by (5). Let $x_{d}(t)$ be a desired signal to be tracked. Therefore, if $\alpha>$ $b \beta>0$, then the control law

$$
u(x, t)=-\alpha \operatorname{sign}\left(\beta \operatorname{sgn}\left(x_{1}-x_{d}\right)+x_{2}-\dot{x}_{d}\right)+b \dot{x}_{d}+\ddot{x}_{d}
$$

satisfies

$$
\lim _{t \rightarrow \infty}\left|x_{1}(t)-x_{d}(t)\right|=0, \quad \lim _{t \rightarrow \infty}\left|x_{2}(t)-\dot{x}_{d}(t)\right|=0 .
$$



Figure 3: Cartesian robot of 3-DOF.

Proof. Let us denote by $e_{1}=x_{1}-x_{d}$ and $e_{2}=x_{2}-\dot{x}_{d}$ the tracking errors. Then their dynamics have the same form as (28). Therefore, by Theorem 5 we obtain that $e_{1} \rightarrow 0$ and $e_{2} \rightarrow 0$, so $x_{1} \rightarrow x_{d}$ and $x_{2} \rightarrow \dot{x}_{d}$ as $t \rightarrow \infty$.

\section{Applications}

In this section we apply the control technique proposed in the previous sections to a pair of systems. To illustrate the complete procedure we consider a 3-DOF Cartesian robot, simulated numerically. Moreover, to show the basic performance of the proposed technique, we present numerical and physical results from the application to a torsional system.

4.1. A Cartesian Robot. A Cartesian robot image and its schematic are presented in Figure 3. The point masses of each link are given by $m_{i}, i=1,2,3$, and the generalized coordinates by $q_{i}, i=1,2,3$; each mass $m_{i}$ and position $q_{i}$ is related by the index. A mathematical model that describes the dynamics of the this system is given by

$$
\left(\begin{array}{ccc}
m_{1}+m_{2}+m_{3} & 0 & 0 \\
0 & m_{2}+m_{3} & 0 \\
0 & 0 & m_{3}
\end{array}\right)\left(\begin{array}{l}
\ddot{q}_{1} \\
\ddot{q}_{2} \\
\ddot{q}_{3}
\end{array}\right)+\left(\begin{array}{c}
0 \\
0 \\
m_{3} g
\end{array}\right)=\left(\begin{array}{l}
\tau_{1} \\
\tau_{2} \\
\tau_{3}
\end{array}\right)
$$

where $g$ is the gravitational component and $\tau_{i}, i=1,2,3$, are the generalized inputs associated with the generalized coordinates.

The system described by (31) can be written as three decoupled subsystems of second-order as follows:

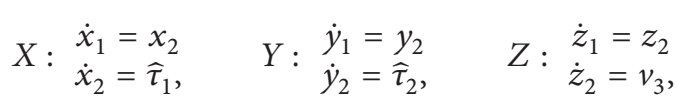

where $x_{1}=q_{1}, x_{2}=\dot{q}_{1}, y_{1}=q_{2}, y_{2}=\dot{q}_{2}, z_{1}=q_{3}, z_{2}=\dot{q}_{3}$, $v_{3}=\widehat{\tau}_{3}-g$, and $\widehat{\tau}_{i}=\tau_{i} / \sum_{j=i}^{3} m_{j}, i=1,2,3$. 


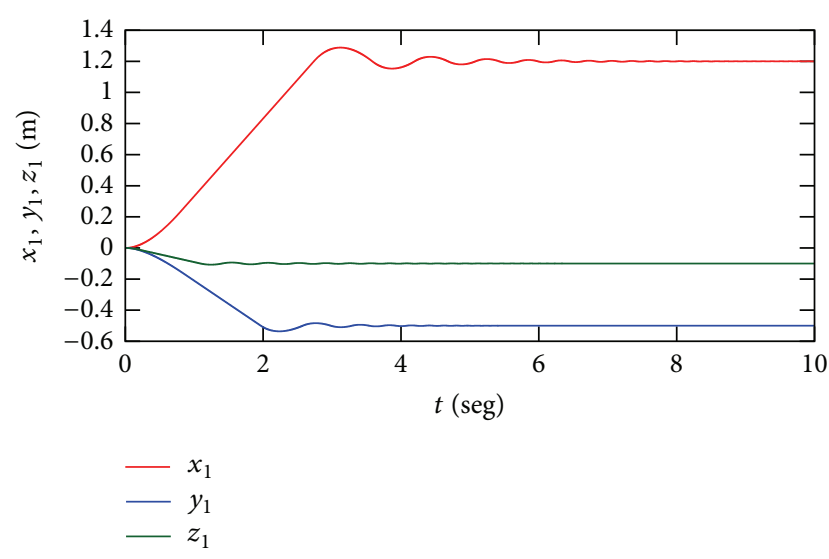

(a)

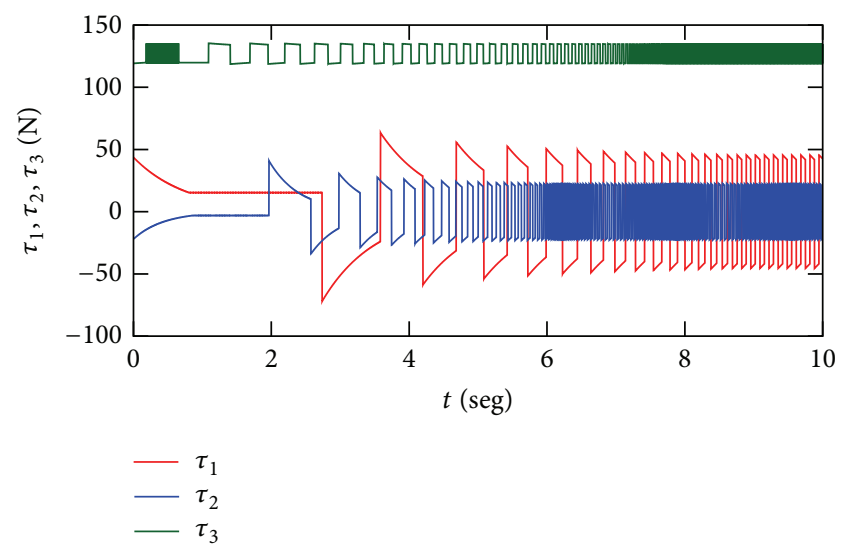

(b)

FIGURE 4: Regulation of the Cartesian robot. (a) Position of generalized coordinates of the controlled Cartesian robot, $x_{1}, y_{1}$, and $z_{1}$. (b) Control signals.

The subsystems (32) are similar to system (5) without viscous friction. Therefore, we can use Corollary 6 to obtain the following control laws:

$$
\begin{aligned}
& \widehat{\tau}_{1}=-b_{1} x_{2}-\alpha_{1} \operatorname{sign}\left(\beta_{1} \operatorname{sgn}\left(x_{1}-x_{d}\right)+x_{2}\right), \\
& \widehat{\tau}_{2}=-b_{2} y_{2}-\alpha_{2} \operatorname{sign}\left(\beta_{2} \operatorname{sgn}\left(y_{1}-y_{d}\right)+y_{2}\right), \\
& v_{3}=-b_{3} z_{2}-\alpha_{3} \operatorname{sign}\left(\beta_{3} \operatorname{sgn}\left(z_{1}-z_{d}\right)+z_{2}\right) .
\end{aligned}
$$

In this case, we add the viscous friction to the controllers. In fact, friction is inherent in all mechanical joint movement and it should be considered in the mathematical model. Then, subsystems $X, Y$, and $Z$ of (32), with the controllers (33), are similar to the system described in Corollary 6. Thus, if the condition imposed by this corollary is satisfied, that is, $\alpha_{i}>b_{i} \beta_{i}, i=1,2,3$, then $\eta \rightarrow \eta_{d}$ and $\dot{\eta} \rightarrow 0$, where $\eta=$ $\left\{x_{1}, y_{1}, z_{1}\right\}$. Finally, the control signals applied to the original system (31) are $\tau_{1}=\left(m_{1}+m_{2}+m_{3}\right) \widehat{\tau}_{1}, \tau_{2}=\left(m_{2}+m_{3}\right) \widehat{\tau}_{2}$, and $\tau_{3}=m_{3}\left(v_{3}+g\right)$.

Example 8 (simulation). Consider the system described by (31) with the control laws given by (33). The parameters used in this example are $m_{1}=16.18, m_{2}=14.562, m_{3}=12.944$, $g=9.81, b_{1}=1.3, b_{2}=2.3$, and $b_{3}=1$. The masses are given in kilograms, the gravity in meters per square seconds, and the coefficients friction by $\mathrm{Ns} / \mathrm{m}$. The controller parameters are given by $\alpha_{1}=1, \alpha_{2}=0.8$, and $\alpha_{3}=0.6 ; \beta_{1}=0.5$, $\beta_{2}=-0.3$, and $\beta_{3}=-0.1$. The desired references are $x_{d}=$ $1.2, y_{d}=-0.5$, and $z_{d}=-0.1$. The initial conditions are $\left(q_{1}, \dot{q}_{1}, q_{2}, \dot{q}_{2}, q_{3}, \dot{q}_{3}\right)=0 \in \mathbb{R}^{6}$ and the simulation time is $t \in[0,10]$.

The positions $x_{1}, y_{1}$, and $z_{1}$ are shown in Figure 4(a). Observe that the positions reach the desired values. The control signals $\tau_{i}, i=1,2,3$, are shown in Figure 4(b).

The phase planes $x_{1}-\dot{x}_{1}, y_{1}-\dot{y}_{1}$, and $z_{1}-\dot{z}_{1}$ are shown in Figures 5(a), 5(b), and 5(c), respectively. In these figures, the horizontal segments correspond to the time interval where the system displays a constant speed. Also, observe that each constant speed has a value given by the parameter $\beta_{i}$. Finally, note that each link position converges to the respective desired positions $x_{d}, y_{d}$, and $z_{d}$.

4.2. A Torsional System. The torsional system (Figure 6) is a simple mechanism described by

$$
J \ddot{q}+\widehat{b} \dot{q}=\widehat{u},
$$

where $J$ is the inertial load, $\widehat{b}$ is the viscous friction coefficient, and the control input $\widehat{u}$ is applied to the main shaft through a DC motor via gears and toothed belts. System output $q$ is the angular position of the inertial load, which is also mechanically connected to the shaft through gears and toothed belts.

By denoting $x_{1}=q$ as the angular position and $x_{2}=\dot{q}$ as the angular velocity, the state equations of system (34) are given by (5), where $b=\widehat{b} / J$ and $u=\widehat{u} / J$, so it is possible to apply the controller given by (8).

To show the effectiveness of the proposed control scheme, some experiments were conducted in the Industrial Emulator ECP220, from Educational Control Products. Numerical and physical experiments were performed, taking into account the information given by the manufacturer. In the physical experiments, the ECP220 was digitally controlled by a computer through an acquisition card; the input to the card is the shaft position, measured by an optical encoder, and the card output is a voltage, which passes through the power source and moves the motor.

From the information provided by the manufacturer we have that the coefficients of inertia and damper are given by $J=0.0206\left(\mathrm{kgm}^{2} / \mathrm{rad}\right)$ and $b=0.05(\mathrm{Nms} / \mathrm{rad})$, respectively. These values were not accurately identified; they were taken directly from the manufacturer manual. Also, the numerical experiments do not include any nonlinear dynamic effect nor parameter uncertainty and assume the availability of the full state (position and velocity).

The coefficients of the controller given in (8) are $\alpha=$ $0.1(\mathrm{Nm})$ and $\beta=1(\mathrm{rad} / \mathrm{s})$. In this case, the control objective 


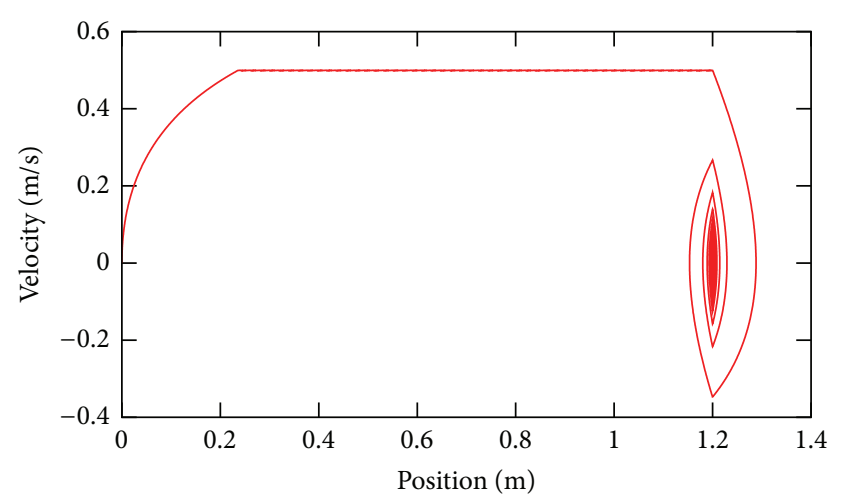

$-X$

(a)

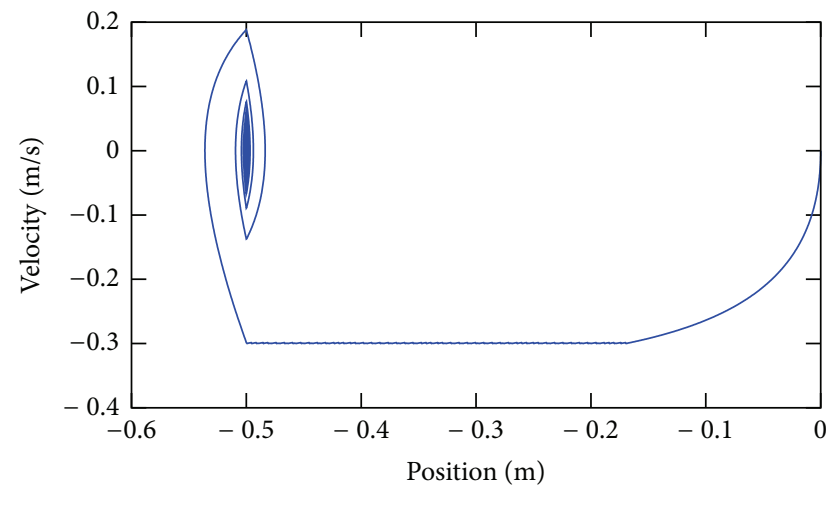

$-Y$

(b)

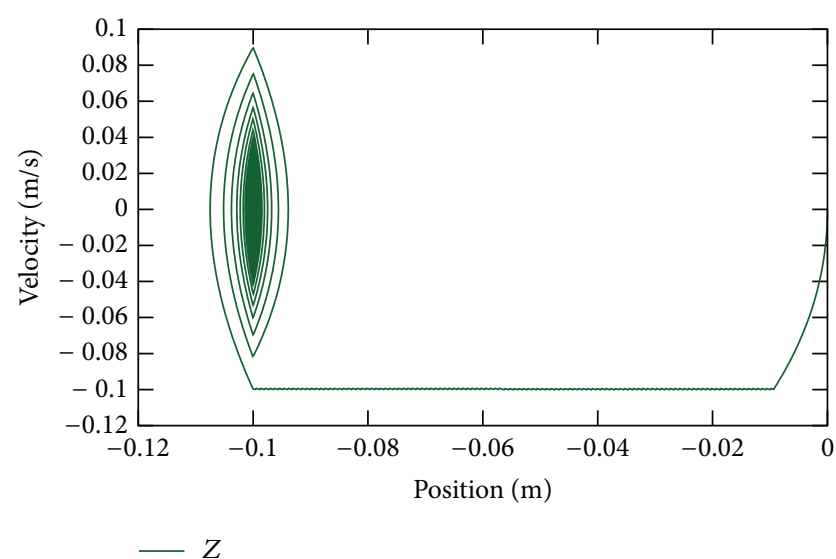

(c)

FIGURE 5: Phase planes of the controlled Cartesian robot.

for velocity is to reach $\pm 1 \mathrm{rad} / \mathrm{s}$ or move at velocities between these values while the position reaches the origin. Note that the cruise speed is given by the parameter $\beta$ of the control design.

Example 9 (simulation). The numerical result of the position is shown in Figure $7(a)$ for $t \in[0,5]$ and the initial conditions

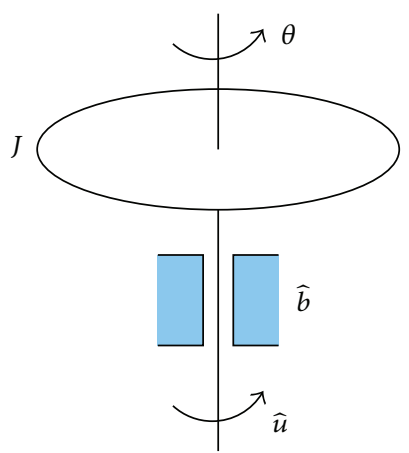

Figure 6: A torsional system.

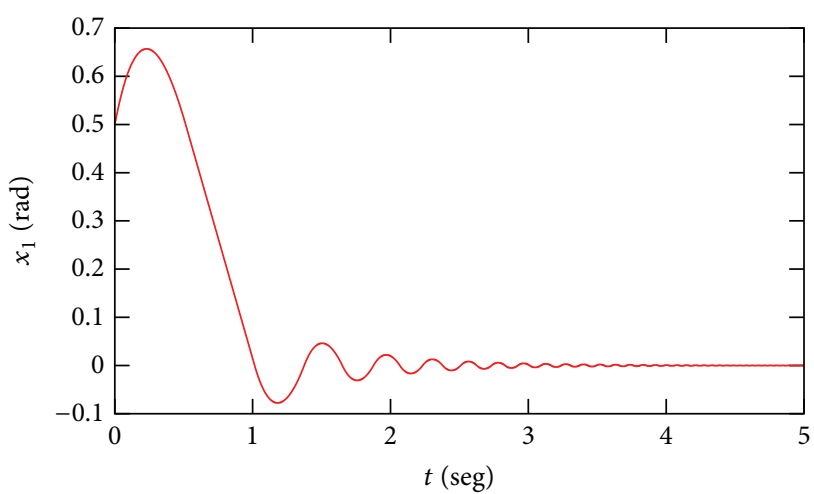

(a)

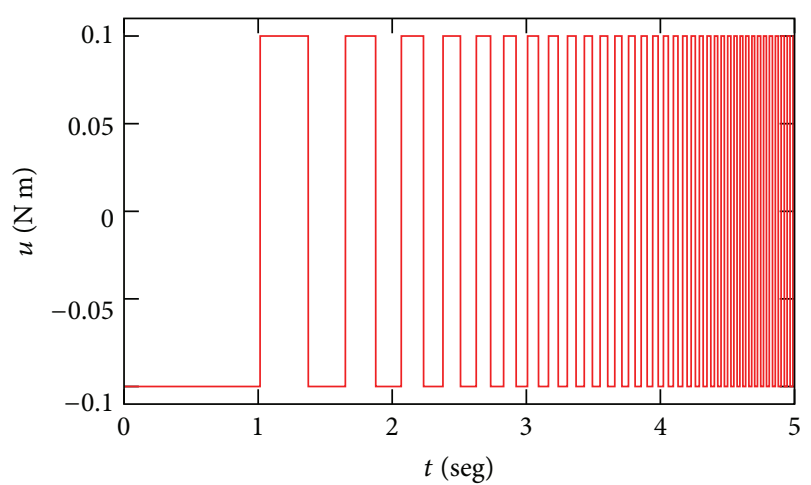

(b)

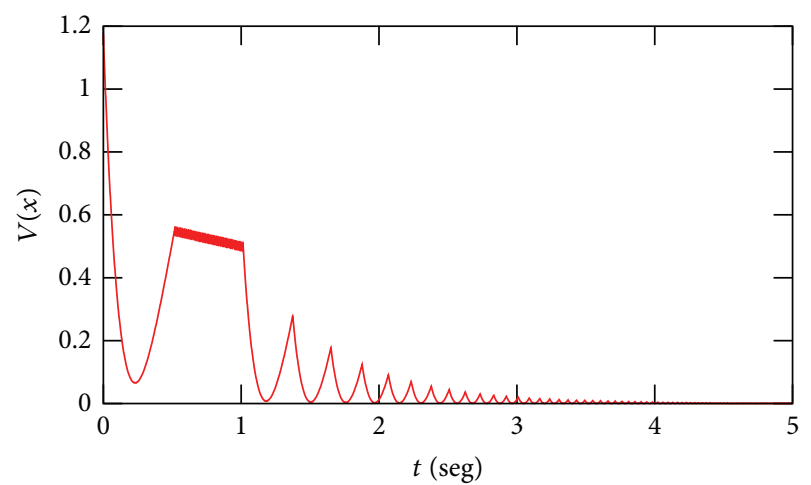

(c)

Figure 7: Numerical results of the controlled torsional system: (a) angular position $x_{1}$, (b) control signal $u$, and (c) the Lyapunov function given in (25). 




FIGURE 8: Numerical phase plane of the controlled torsional system.

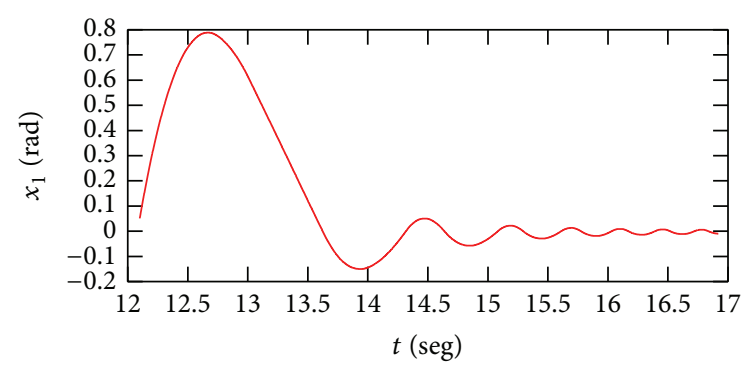

(a)

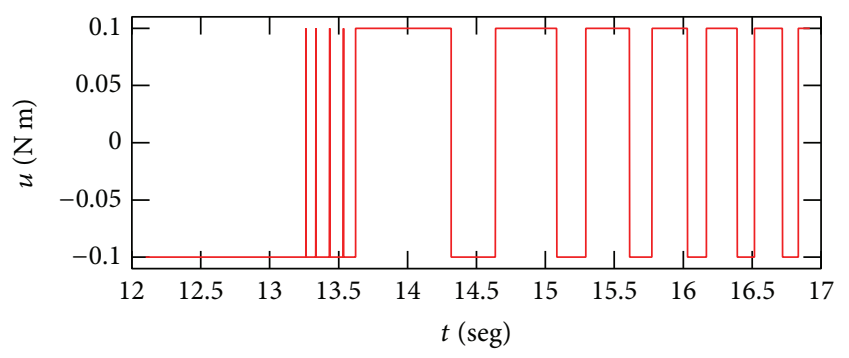

(b)

Figure 9: Experimental results of the controlled torsional system: (a) angular position $x_{1}$; (b) control signal $u$.

$x_{1}(0)=0.5 \mathrm{rad}$ and $x_{2}(0)=1.5 \mathrm{rad} / \mathrm{s}$. Meanwhile, the control signal is shown in Figure 7(b). Figure 7(c) shows the time evolution of the Lyapunov function given in (4). Observe that the peaks occur when the solution crosses over the $x_{2}$-axis, that is, at each crossing on the surface of commutation the value of $V(x)$ is lower than the previous crossing. The Lyapunov function is zero when the variable state arrives at the origin. Finally, Figure 8 shows the numerical results of the state when the initial conditions are given by $x(0) \in X_{1} \times X_{2}$, where $X_{1}=\{-2,-1,0,1,2\}$ and $X_{2}=\{-1.5,1.5\}$.

Example 10 (experimental regulation). To implement physically the controller proposed in (8), the equipment ECP220 was used. To estimate the speed, we employed a linear observer with gain $K=\left[122.57,3.45 \times 10^{3}\right]^{T}$. Figure 9 shows the angular position and the control signal, while Figure 10 shows the experimental results in the phase plane $x_{1}-x_{2}$, along with other experiments at different initial conditions.

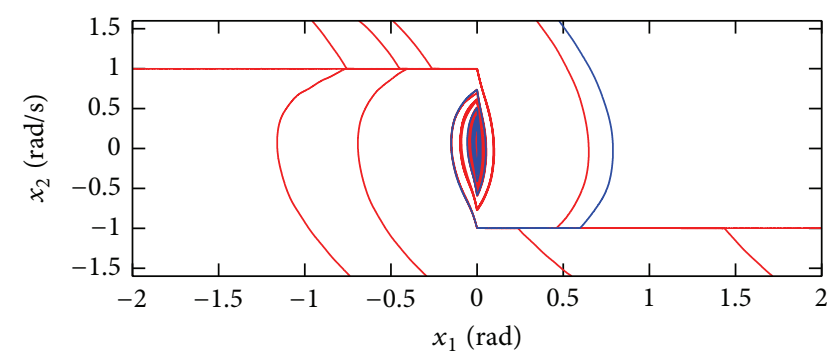

FIGURE 10: Experimental phase plane of the controlled torsional system.

From Figures 8 and 10, we observe a remarkable similitude of the phase planes, even when the parameter values were approximated, and the presence of nonlinearities in the real system, like backlash in the belts and Coulomb friction, among other physical phenomena, which were not considered. The control objectives are accomplished; that is, the system moves over an imposed velocity, while it arrives to the final desired position, in an exponential way.

Finally, observe that all solutions that leave the point $(0, \pm \beta)$ have identical evolution in time; therefore, the convergence to the origin is identical.

Example 11 (experimental tracking). In Figure 11(a) we illustrate the application of Corollary 7. In this case, the reference $x_{d}$ is given by a cosine function whose amplitude and frequency are one. In the first seconds we can observe a difference between the reference and the angular position of the ECP220 (mainly due to the nonmodeled dry friction); however, this difference decreases significantly after a short transient.

The first and second derivatives of the desired position are precalculated to be used by the control law (29) (see Figure 11(b)). The considerations and the other parameters were the same as given in the previous example. The error signals between the reference and position are shown in Figure 11(c).

\section{Conclusions}

We have proposed a discontinuous controller for mechanical systems with the objective to regulate the position and maintain, as long as possible, the speed at a specified value until the system is close to the desired position. This controller can be applied to robotic systems whose degrees of freedom are, or can be, decoupled. In fact, due to the robustness inherited from sliding modes, the same controller can be applied also to robotic systems with weakly coupled degrees of freedom.

The proposed control technique has been applied to a Cartesian robot, simulated numerically, and a real rotational system that emulates the basic dynamics of a large class of robotic systems. It can be applied also to mobile robots, SCARA robots, and other important systems. 


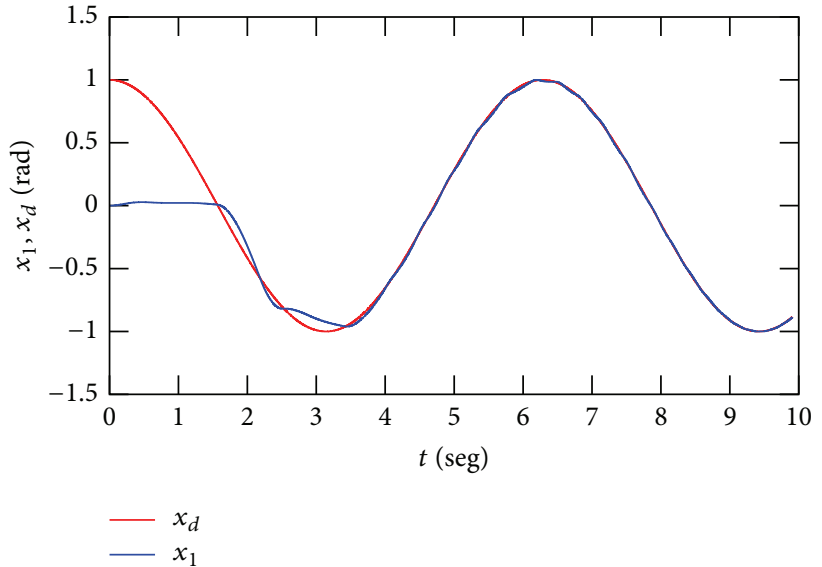

(a)

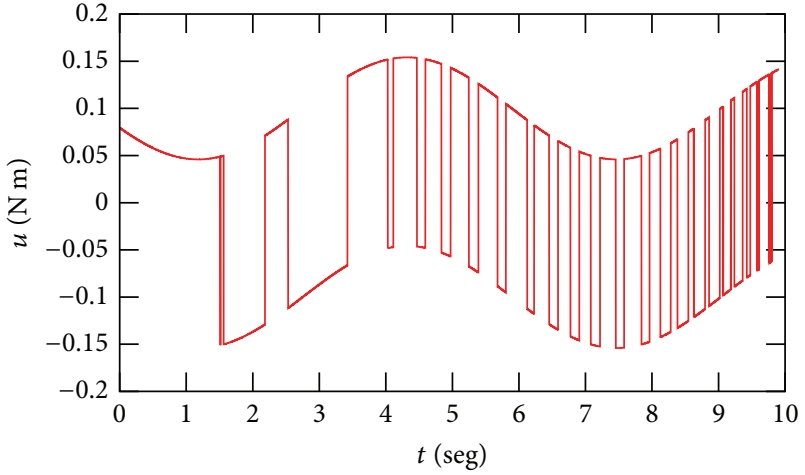

(b)

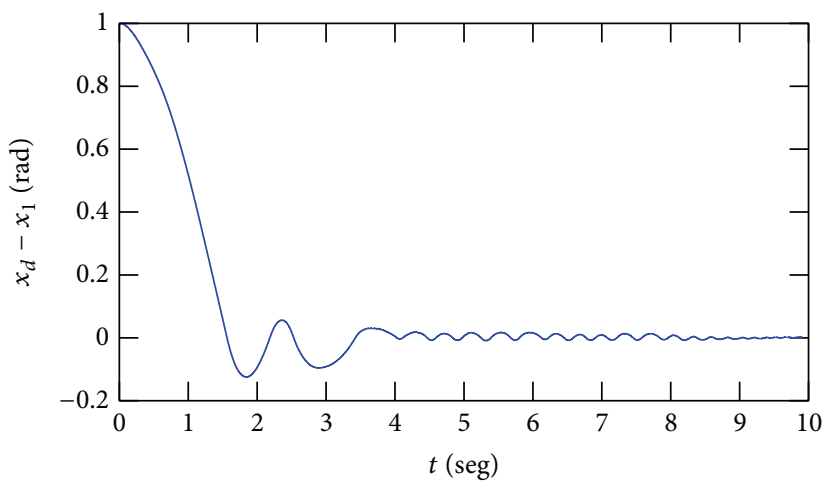

(c)

FIGURE 11: Experimental tracking of the controlled torsional system: (a) angular position $x_{1}$ and reference $x_{d}$; (b) control signal $u$; (c) error signal $x_{d}-x_{1}$.

\section{Conflict of Interests}

The authors declare that there is no conflict of interests regarding the publication of this paper.

\section{Acknowledgments}

The equipment ECP-220 was borrowed from the Control Laboratory in CICESE. This work was partially supported by the National Council of Science and Technology (CONACYT) of Mexico, under Grant no. CB-2012-01-180011.

\section{References}

[1] Y. Feng, X. Yu, and F. Han, "On nonsingular terminal slidingmode control of nonlinear systems," Automatica, vol. 49, no. 6 , pp. 1715-1722, 2013.

[2] Y. Feng, F. Han, and X. Yu, "Chattering free full-order slidingmode control," Automatica, vol. 50, no. 4, pp. 1310-1314, 2014.

[3] F. Barrero, A. González, A. Torralba, E. Galván, and L. G. Franquelo, "Speed control of induction motors using a novel fuzzy sliding-mode structure," IEEE Transactions on Fuzzy Systems, vol. 10, no. 3, pp. 375-383, 2002.

[4] E. Y. Y. Ho and P. C. Sen, "Control dynamics of speed drive systems using sliding mode controllers with integral compensation," IEEE Transactions on Industry Applications, vol. 27, no. 5, pp. 883-892, 1991.
[5] S. M. Loos, A. Platzer, and L. Nistor, "Adaptive cruise control: hybrid, distributed, and now formally verified," in FM 2011: Formal Methods, vol. 6664 of Lecture Notes in Computer Science, pp. 42-56, Springer, Berlin, Germany, 2011.

[6] V. Carrara, R. H. Siqueira, and D. Oliveira, "Speed and current control mode strategy comparison in satellite attitude control with reaction wheels," in Proceedings of the 21st Brazilian Congress of Mechanical Engineering (COBEM '11), Natal, Brazil, 2011.

[7] Y. S. Chati and H. Balakrishnan, "Aircraft engine performance study using flight data recorder archives," in Proceedings of the Aviation Technology, Integration, and Operations Conference, Los Angeles, Calif, USA, August 2013.

[8] A. Sengupta, M. Pauken, A. Kennett, M. Trinidad, and E. Zabrensky, "Systems engineering and support systems technology considerations of a mars ascent vehicle," in Proceedings of the IEEE Aerospace Conference, pp. 1-11, Big Sky, Mont, USA, March 2012.

[9] T. Shima, M. Idan, and O. M. Golan, "Sliding-mode control for integrated missile autopilot guidance," Journal of Guidance, Control, and Dynamics, vol. 29, no. 2, pp. 250-260, 2006.

[10] X. Rong and U. Ozguner, "Sliding mode control of a quadrotor helicopter," in Proceedings of the 45th IEEE Conference on Decision and Control, pp. 4957-4962, IEEE, San Diego, Calif, USA, December 2006.

[11] L. Wang, H. Xu, and H. Luo, "An intelligent cruise controller for high-speed train operation based on fuzzy neural network 
theory," Applied Mechanics and Materials, vol. 300-301, pp. 1405-1411, 2013.

[12] S. Wang, H.-W. Li, H.-R. Meng, and Q.-L. Wu, "Active disturbance rejection controller for speed-loop in telescope servo system," Optics and Precision Engineering, vol. 19, no. 10, pp. 2442-2449, 2011.

[13] H. J. Xie, D. L. Kerstetter, and A. S. Mattila, "The attributes of a cruise ship that influence the decision making of cruisers and potential cruisers," International Journal of Hospitality Management, vol. 31, no. 1, pp. 152-159, 2012.

[14] J. Alvarez, I. Orlov, and L. Acho, "An invariance principle for discontinuous dynamic systems with application to a Coulomb friction oscillator," Journal of Dynamic Systems, Measurement and Control, Transactions of the ASME, vol. 122, no. 4, pp. 687690, 2000. 


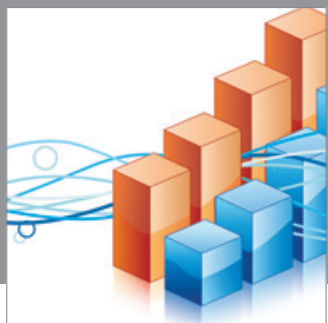

Advances in

Operations Research

mansans

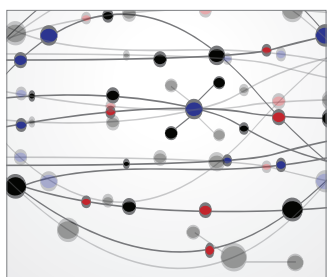

The Scientific World Journal
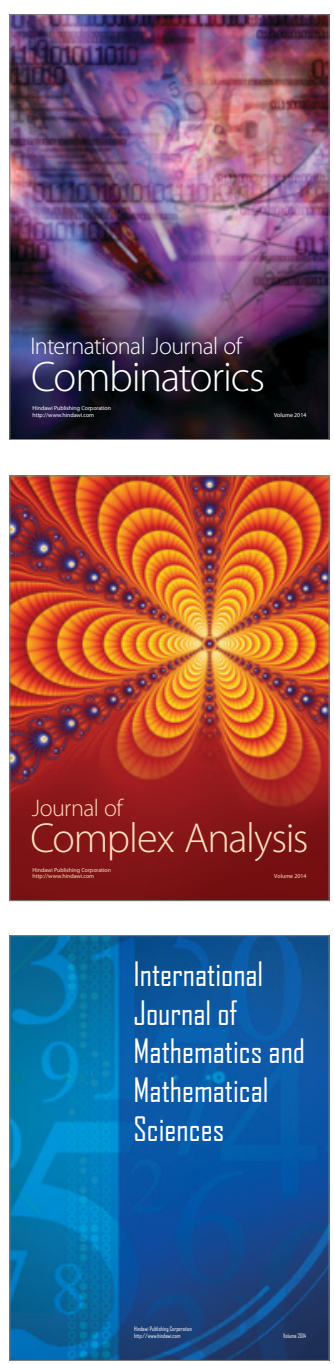
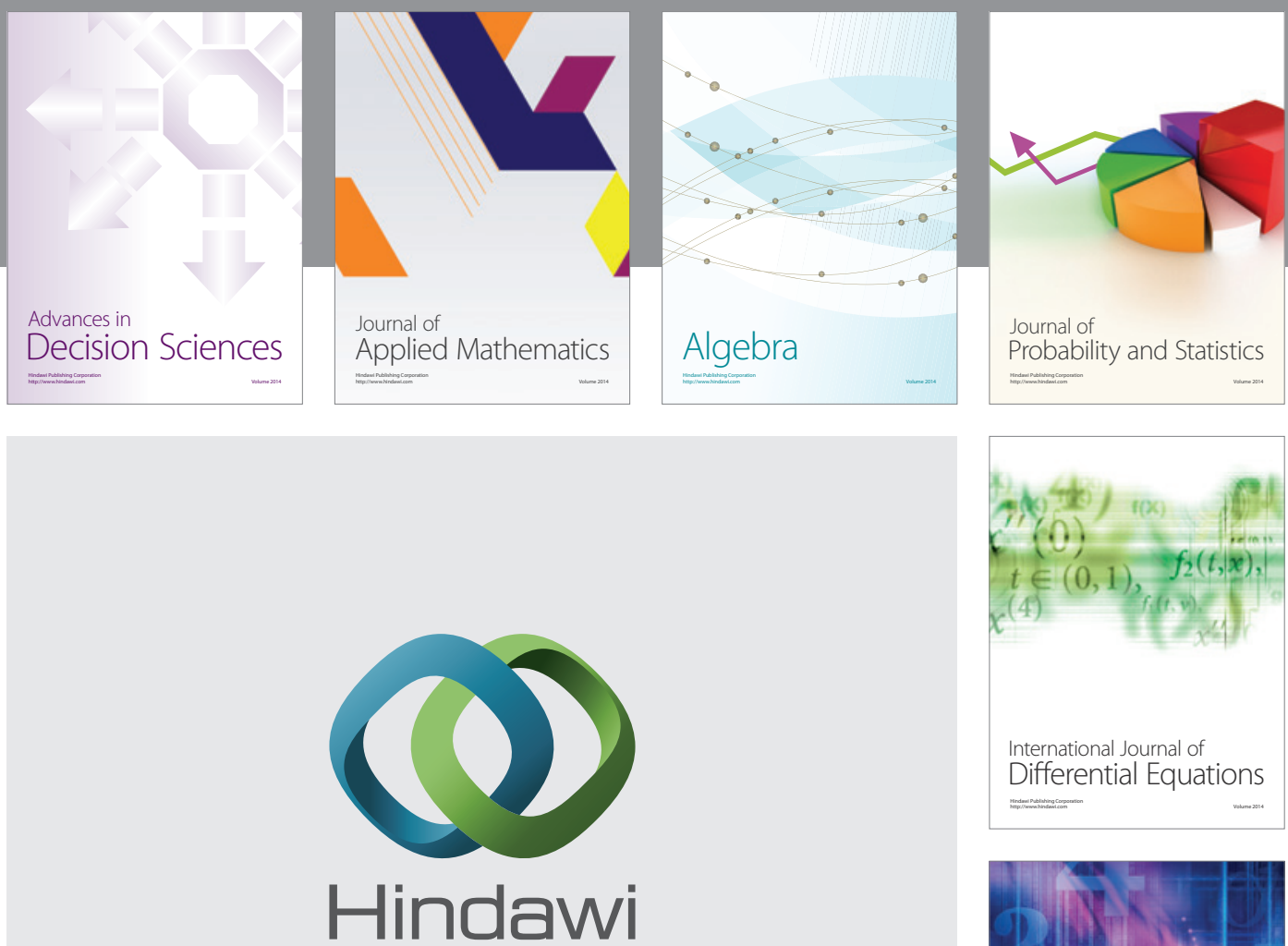

Submit your manuscripts at http://www.hindawi.com
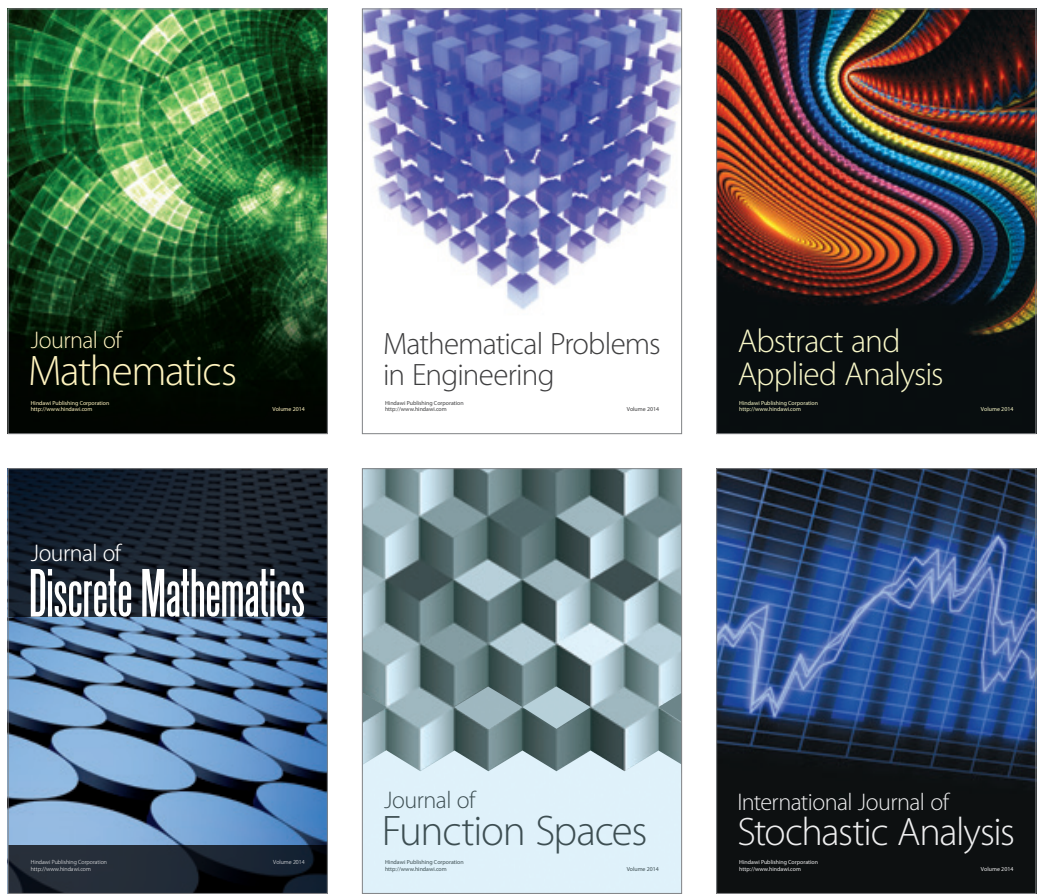

Journal of

Function Spaces

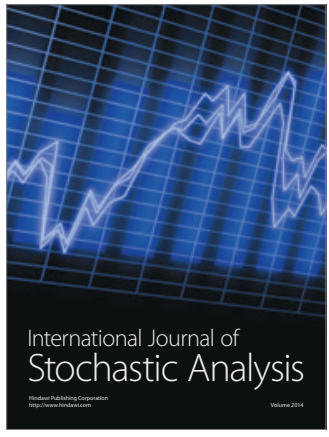

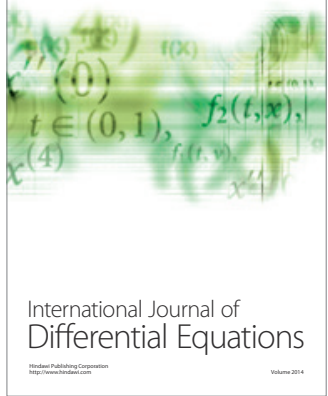
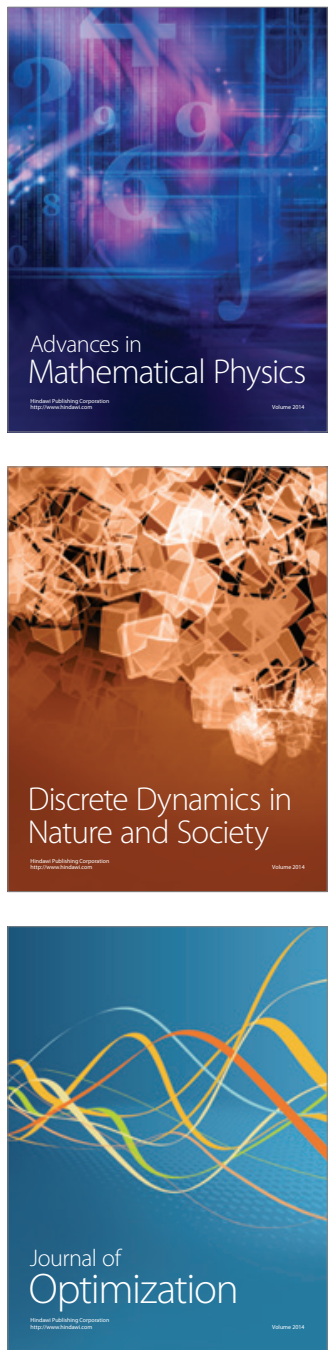